\title{
Osteoblast proliferation correlates with cell morphology on dental implants and is modulated by surface parameters
}

Kerstin Rabel ${ }_{1}$, Ralf-Joachim Kohal, $\mathrm{PhD}_{1}, \mathrm{DMD}$, Thorsten Steinberg, $\mathrm{PhD}_{2}$, Erik Adolfsson, $\mathrm{PhD}_{4}$, Paola Palmero, $\mathrm{PhD}_{5}$, Tobias Fürderer ${ }_{6}$, Brigitte Altmann, $\mathrm{PhD}_{3}$

${ }_{1}$ Department of Prosthodontic Dentistry, 2 Department of Operative Dentistry and Periodontology, Division of Oral Biotechnology, 3 Department of Oral and Maxillofacial Surgery, University Medical Center Freiburg, Freiburg, Germany, ${ }_{4}$ Swerea IVF AB, Mölndal, Sweden, ${ }_{5}$ Politecnico di Torino, Torino 10129, Italy, ${ }_{6}$ DOCERAM Medical Ceramics GmbH

Dortmund, Germany

\section{Background and Aim}

Since physicochemical properties of oral implant surfaces are crucial for implant success by influencing cell responses at biomaterial interfaces ${ }^{1,2}$, current research focuses on characterizing the modulation of cell functions by implant surface attributes to improve the healing process.

The present study aimed at examining the early response of primary human osteoblasts derived from alveolar bone $(\mathrm{AO})$ to zirconia-based yttria- and ceria-supplemented biomaterials ( $\mathrm{Y}$ /Ce-TZP) with different surface modifications by examining cell attachment, morphology and proliferation.

\section{Methods and Materials}

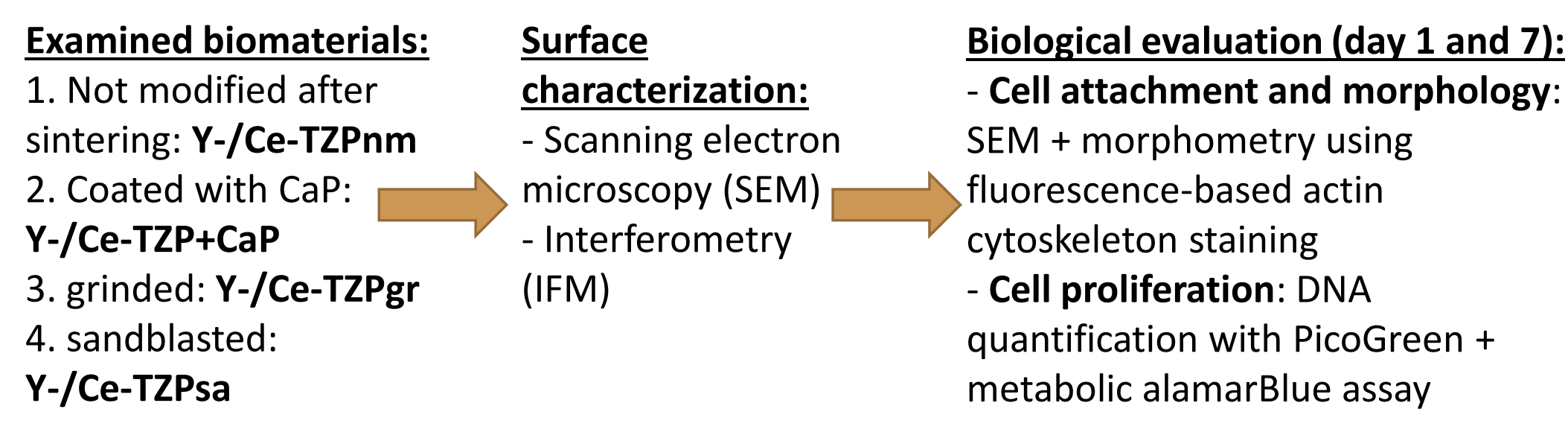

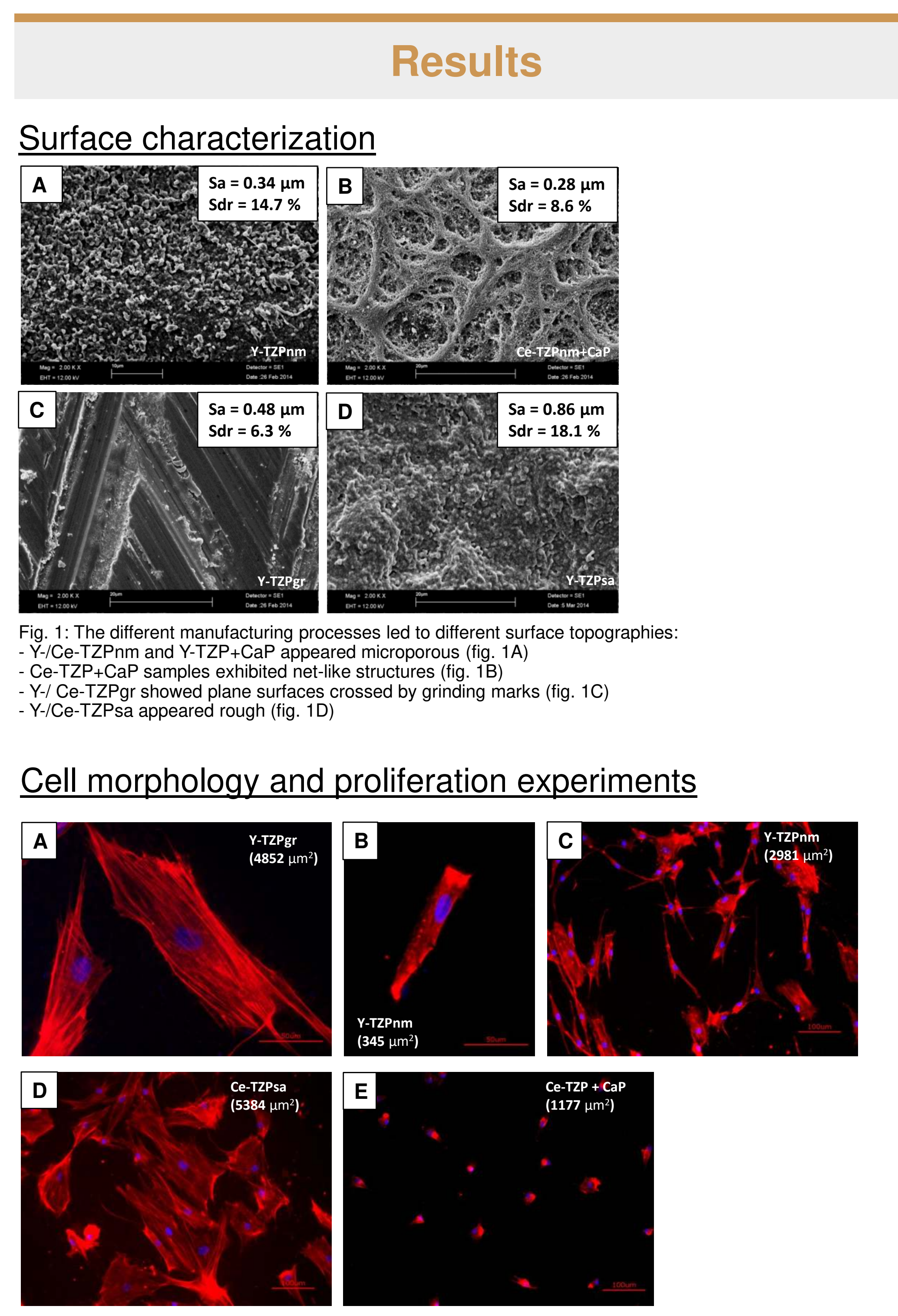

Fig. 2: Representative micrographs of fluorescence based actin staining taken at day 1 (fig. $2 \mathrm{~A}, \mathrm{~B})$ and 7 (fig.
$2 \mathrm{C}-\mathrm{E})$ showing rectangular $\mathrm{AO}$ on grinded surfaces (fig. $2 \mathrm{~A}$ ), elongated $\mathrm{AO}$ on all other surfaces (fig. $2 \mathrm{~B}$ ),

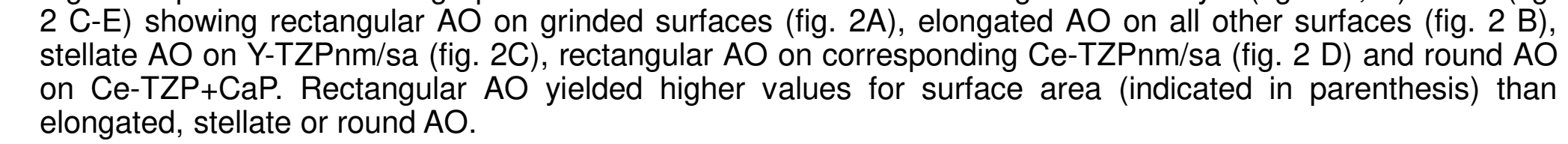$$
\text { A }
$$

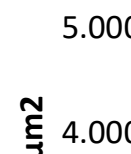
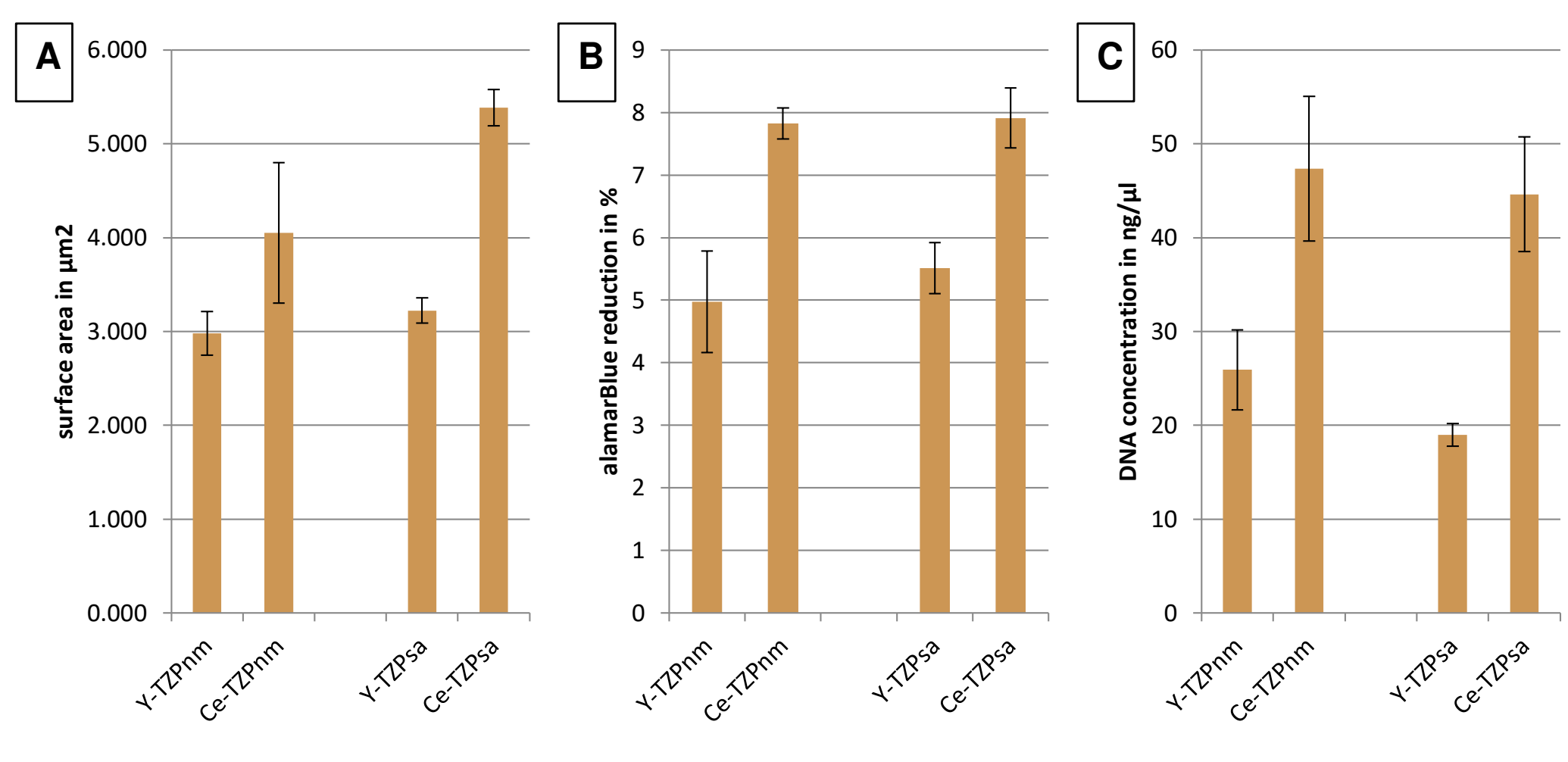

Fig. 4: Excerpt of the results obtained at day 7 via morphometric analysis (A), alamarBlue assay (B) and DNA concentration $(C)$ showing that all of these parameters were favored by Ce-TZP surfaces in comparison to $Y$ -
TZP samples. Differences between Ce- and $\mathrm{Y}$-TZP surfaces reached statistical significance $(\mathrm{p}<0.05)$ for TZP samples. Differences between Ce- and Y-TZP surfaces reached
alamarBlue reduction (Tukey's HSD test) and surface area (Dunn's test).
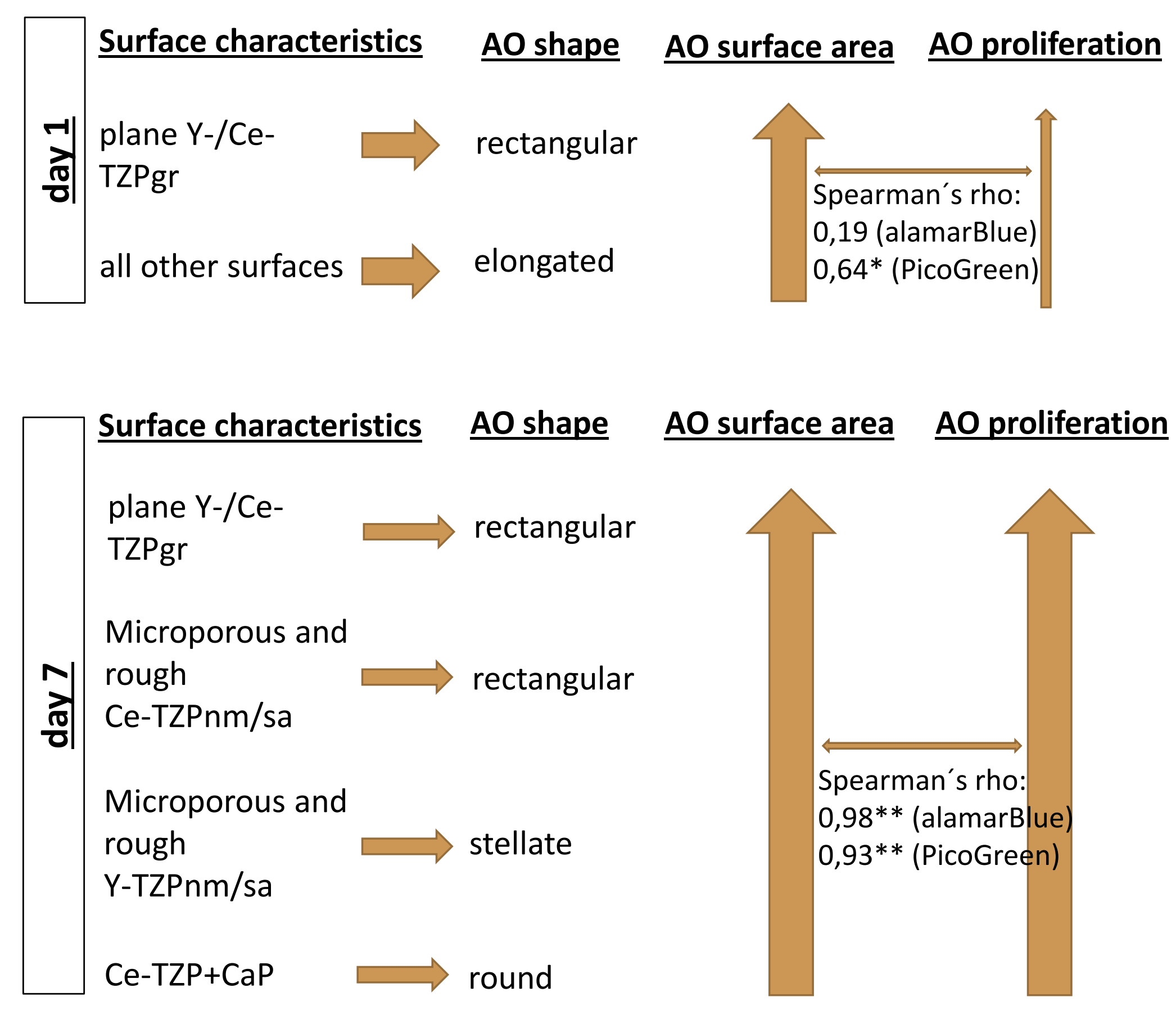

Fig. 5: Summary of the results obtained by analysis of cell morphology and proliferation. Values obtained via Spearman's correlation test for interdependence of surface area and proliferation parameters are listed. Via
Spearman's test values ranging from -1 to 1 can be calculated while values close to -1 suggest an inverse correlation, values around 0 reject any correlation and values close to 1 point at strong correlation between

Biological evaluation revealed that:

- AO functions at day 1 were mainly modulated by surface topography

- at day 7 AO functions were determined mainly by surface chemistry

- AO proliferation was strongly dependent on AO morphology at day 7

- AO showed a switch in their proliferation behavior from low to high proliferation by exceeding a critical cell size

\section{Conclusions}

We could demonstrate that $A O$ morphology and proliferation are influenced in a biomaterial- and time-dependent manner. Furthermore, we could show that AO proliferation is a function of AO morphology and we therefore conclude that cellular behavior on oral implant surfaces can be influenced by designing surfaces in that way that they induce cell morphologies favoring cell proliferation which in turn may lead to an improved implant healing course.

\section{References}

- Altmann, B. et al. (2013). Distinct cell functions of osteoblasts on UV-functionalized titanium- and zirconia-based implant materials are modulated by surface topography. Tissue Engineering Part C: Methods 19(11): 850-863.

2. Anselme, K., et al.. (2010). Relative influence of surface topography and surface chemistry on cell response to bone implant materials. Part 2: biological aspects. Proceedings of the Institution
Medicine, 224(12), 1487-1507.

The research was supported by the European Community's Seventh Framework Program (grant agreement n. 280741). 